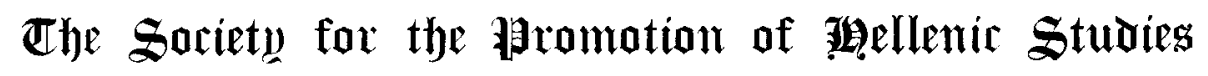

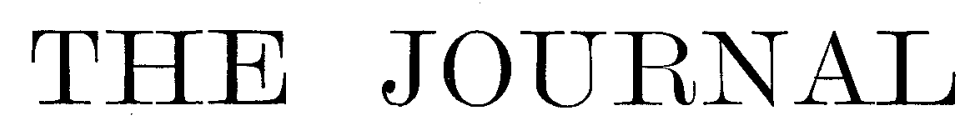

oF

\title{
HELLENIC STUDIES
}

VOLUME XIII. (I892-3)

PUBLISHED BY THE COUNCIL, AND SOLD ON THEIR BEHAIF

BY

MACMILLAN AND CO., 29 BEDFORD STREET, STRAND

LONDON

MDCCOXCIII

The Rights of Translation and Reproduction are Reserved, 
Ricitard Clay and Sons, Limited LONDON AND BUNGAY. 


\section{CONTENTS.}

Rules of the Society

$\begin{array}{llllllllll}\text { List of Officers and Members } & \ldots & \ldots & \ldots & \ldots & \ldots & \ldots & \ldots & \ldots & \text { xv }\end{array}$

Additions to Library ...

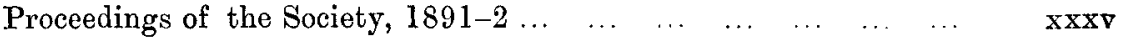

$\begin{array}{lllllllll}1892-3 & \ldots & \ldots & \ldots & \ldots & \ldots & \ldots & \ldots & \mathrm{xl}\end{array}$

1. Three Attic Lekythoi from Eretria. (Plates I. -III.)--Eugénie Sellers 1

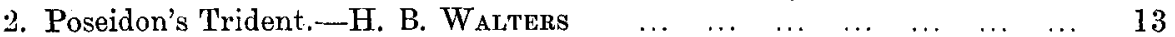

3. Palladia from Mycenae. —E. A. Gardner $\ldots \begin{array}{llllllll}\ldots & \ldots & \ldots & \ldots & \ldots & \ldots & \ldots & 21\end{array}$

4. Iron in Homer.-F. B. Jevons $\ldots$..

5. On the Ancient Hecatompedon which occupied the Site of the Parthenon

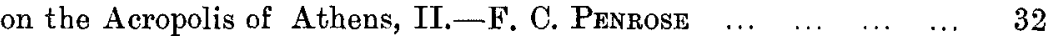

6. The Procedure of the Gortynian Inscription.—J. W. Headla

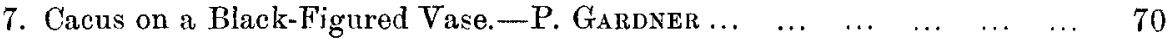

8. Odysseus and Kirke on a Boeotian Vase. (Plate IV.) - H. B. Walters 77

9. Recent Additions to the Sculptures of the Parthenon. (Plate V.)-

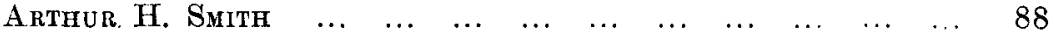

10. The Leper Terra-Cotta of Athens.-Francrs E. Hoggan, M.D. $\ldots \quad \ldots \quad 101$

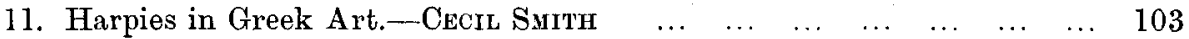

12. Deme Legends on Attic Vases.-Cerric Smith $\ldots \begin{array}{lllllll}\ldots & \ldots & \ldots & \ldots & \ldots & \ldots & 115\end{array}$

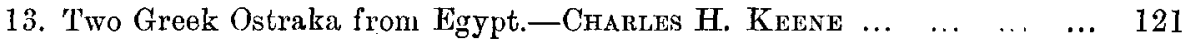

14. The Bronze Fragments of the Acropolis: I. (Plates VI.--VII.)-A. G.

$\begin{array}{llllllllllllll}\text { BATHER... } & \ldots & \ldots & \ldots & \ldots & \ldots & \ldots & \ldots & \ldots & \ldots & \ldots & \ldots & \ldots & 124\end{array}$

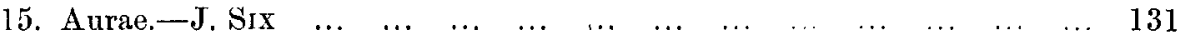

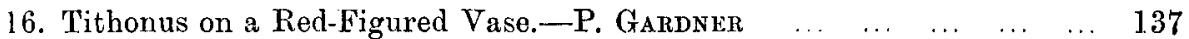

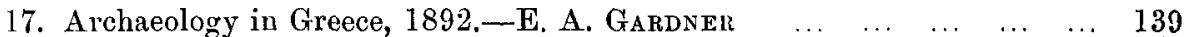

18. The Pre-Persian Temple on the Acropolis. - J. G. Frazer $\quad \ldots \quad \ldots \quad \ldots \quad 154$

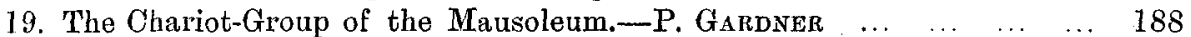

20. A Mykênaean Treasure from Aegina.-A. J. Evans $\quad \ldots \quad \ldots \quad \ldots \quad \ldots \quad 195$

21. Excavations on the Probable Sites of Basilis and Bathos.-A. G. Bather $\begin{array}{lllllllllllll}\text { and } \mathrm{V} . \mathrm{W} . \text { YorKE } & \ldots & \ldots & \ldots & \ldots & \ldots & \ldots & \ldots & \ldots & \ldots & \ldots & \ldots & 227\end{array}$

22. The Bronze Fragments of the Acropolis: II. (Plates VIII., IX.).-

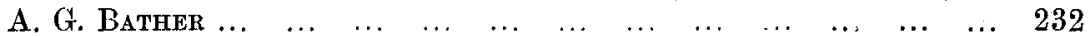

23. Newly Discovered Fragments of the Balustrade of Athena Nike.

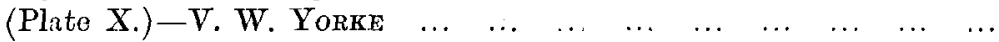

24. Selected Vase Fragments from the Acropolis of Athens: I. (Plates

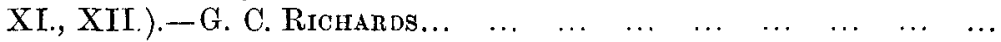

25. Waxen Tablets with Fables of Babrius (Tabulae Ceratae Assendelftianae). (Plates XIII.—XIX.).—D. C. Hessedriva $\ldots \begin{array}{lllll}\ldots & \ldots & \ldots & \ldots & 293\end{array}$

26. A Terra-Cotta Antefix from Lanuvium.-_- A. S. Murray ... $\quad \ldots \quad \ldots \quad \ldots \quad 315$

27. The Thersilion at Megalopolis. (Plate XXI.).--E. F. Benson $\quad \ldots \quad \ldots \quad 319$

28. The Development of the Plan of the Thersilion.-A. G. BATHER... . . 328

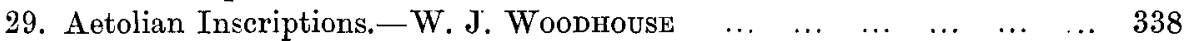

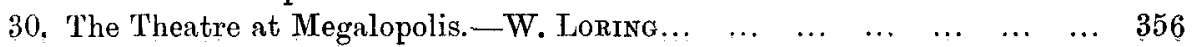




\section{LIST OF PLATES.}

I. Attic Lekythos from Fretria : Odysseus and the Sirens.

II. , , , , , , Kirke.

III. " , " , , , Herakles and Atlas.

IV. Odysseus and Kirke on a Boeotian Vase.

V. Group from the West Pediment of the Parthenon.

VI., VII. Inscribed Bronzes from the Acropolis, Athens.

VIII. Bronze Fragment from the Acropolis, Athens.

IX. Bronze Fragments from Eleutherae.

X. A New Fragment of the Nike Balustrade, Athens.

XI., XII. Fragments of Vases from the Acropolis, Athens.

XIII., XIV., XV., XVI., XVII., XVIII., XIX. Wax Tablets containing Fables of Babrius.

XXI. Plan of the Thersilion, Megalopolis.

NOTE.

Owing to an error, there is no Plate XX. issued with the present number. 


\section{LIST OF WOOD-CUTS, \&.}

Forms of Lekythoi $\ldots \begin{array}{lllllllllllll} & \ldots & \ldots & \ldots & \ldots & \ldots & \ldots & \ldots & \ldots & \ldots & \ldots & \ldots & 2\end{array}$

Head of Odysseus from Lekgthos, Restored $\ldots \ldots \begin{array}{llllllll}\ldots & \ldots & \ldots & \ldots & \ldots & \ldots & \ldots & 7\end{array}$

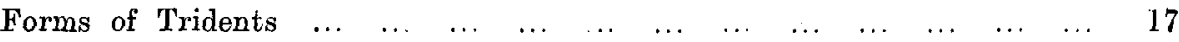

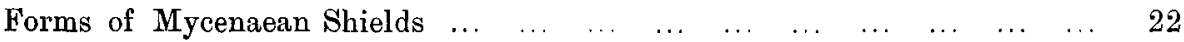

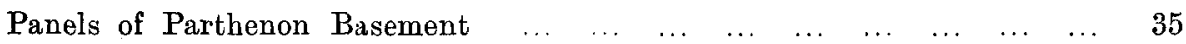

$\begin{array}{llllllllllllll}\text { Profiles of Stylobate Courses } & \ldots & \ldots & \ldots & \ldots & \ldots & \ldots & \ldots & \ldots & \ldots & \ldots & 36\end{array}$

Foundations of Hecatompedon $\quad \ldots \begin{array}{lllllllllll} & \ldots & \ldots & \ldots & \ldots & \ldots & \ldots & \ldots & \ldots & 40\end{array}$

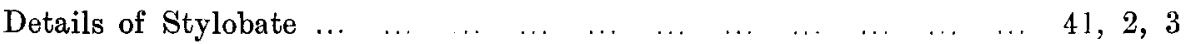

$\begin{array}{lllllllllllllll}\text { Marks on Stylobate } & \ldots & \ldots & \ldots & \ldots & \ldots & \ldots & \ldots & \ldots & \ldots & \ldots & \ldots & \ldots & 45\end{array}$

Curvature of Horizontal Lines of Parthenon $\ldots \begin{array}{lllllllll}\ldots & \ldots & \ldots & \ldots & \ldots & \ldots & \ldots & 46\end{array}$

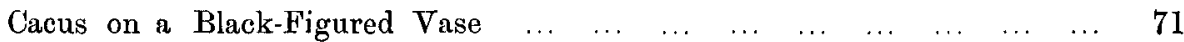

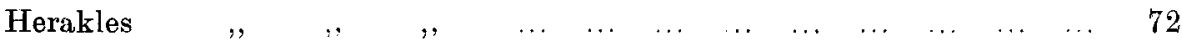

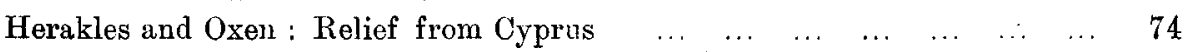

$\begin{array}{llllllllllllllll}\text { Boeotian Vase } & \ldots & \ldots & \ldots & \ldots & \ldots & \ldots & \ldots & \ldots & \ldots & \ldots & \ldots & \ldots & \ldots & 78\end{array}$

Odysseus and Kirke : Van Branteghem Vase $\ldots \begin{array}{lllllllll}\ldots & \ldots & \ldots & \ldots & \ldots & \ldots & \ldots & 81\end{array}$

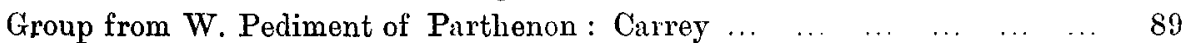

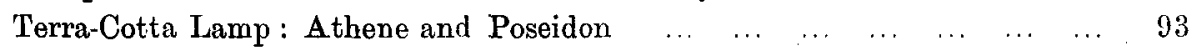

Head of Lapith $\ldots \begin{array}{llllllllllllll} & \ldots & \ldots & \ldots & \ldots & \ldots & \ldots & \ldots & \ldots & \ldots & \ldots & \ldots & \ldots & 94\end{array}$

Group from N. Frieze of Parthenon : Carrey $\ldots \begin{array}{lllllllll}\ldots & \ldots & \ldots & \ldots & \ldots & \ldots & \ldots & 96\end{array}$

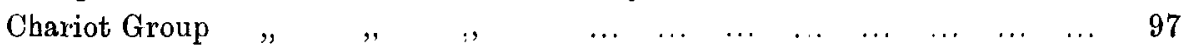

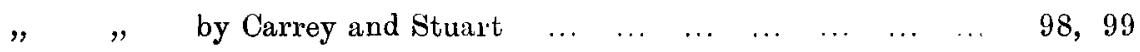

Group from N. Frieze... $\ldots \begin{array}{llllllllllllll} & \ldots & \ldots & \ldots & \ldots & \ldots & \ldots & \ldots & \ldots & \ldots & \ldots & \ldots & 100\end{array}$

$\begin{array}{lllllllllllll}\text { Terra-Cotta representing a Lepel } & \ldots & \ldots & \ldots & \ldots & \ldots & \ldots & \ldots & \ldots & \ldots & 101\end{array}$

$\begin{array}{llllllllllll}\text { Winged Figure (from situla) } & \ldots & \ldots & \ldots & \ldots & \ldots & \ldots & \ldots & \ldots & \ldots & \ldots & 109\end{array}$

Europa: Vase from Caere $\ldots \begin{array}{lllllllllllll}\ldots & \ldots & \ldots & \ldots & \ldots & \ldots & \ldots & \ldots & \ldots & \ldots & \ldots & 112\end{array}$

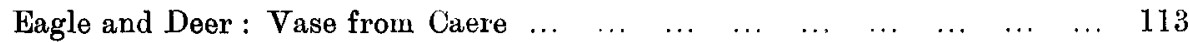

$\begin{array}{llllllllllllll}\text { Vulture and Crow } & \ldots & \ldots & \ldots & \ldots & \ldots & \ldots & \ldots & \ldots & \ldots & \ldots & \ldots & \ldots & 114\end{array}$

$\begin{array}{lllllllllllll}\text { Amphora from Vulci } \ldots & \ldots & \ldots & \ldots & \ldots & \ldots & \ldots & \ldots & \ldots & \ldots & \ldots & \ldots & 116\end{array}$

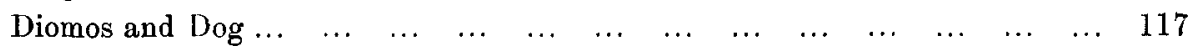

Inscribed Ostraka from Egypt... 
Fist:

$\begin{array}{llllllllllll}\text { Figures from Astragalos Vase } & \ldots & \ldots & \ldots & \ldots & \ldots & \ldots & \ldots & \ldots & \ldots & \ldots & 135\end{array}$

$\begin{array}{llllllllllllll}\text { Tithonus and Eos } & . & \ldots & \ldots & \ldots & \ldots & \ldots & \ldots & \ldots & \ldots & \ldots & \ldots & \ldots & 137\end{array}$

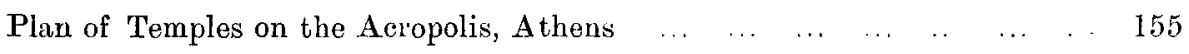

Treasure from Aegina-

$\begin{array}{lllllllllllllll}\text { Gold Cup } & \ldots & \ldots & \ldots & \ldots & \ldots & \ldots & \ldots & \ldots & \ldots & \ldots & \ldots & \ldots & \ldots & 196\end{array}$

$\begin{array}{lllllllllllll}\text { Pendant Ornament } & \ldots & \ldots & \ldots & \ldots & \ldots & \ldots & \ldots & \ldots & \ldots & \ldots & \ldots & 197\end{array}$

Types from Nineveh and Italy $\ldots \begin{array}{llllllllll}\ldots & \ldots & \ldots & \ldots & \ldots & \ldots & \ldots & \ldots & 201,202\end{array}$

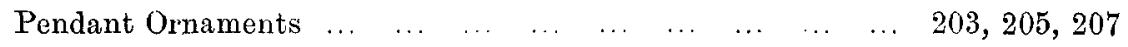

$\begin{array}{llllllllllllll}\text { Necklaces } & \ldots & \ldots & \ldots & \ldots & \ldots & \ldots & \ldots & \ldots & \ldots & \ldots & \ldots & \ldots & 208,209\end{array}$

$\begin{array}{llllllllllll}\text { Plates and Diadems } & \ldots & \ldots & \ldots & \ldots & \ldots & \ldots & \ldots & \ldots & \ldots & \ldots & 210,211\end{array}$

$\begin{array}{lllllllllllll}\text { Finger Rings } & \ldots & \ldots & \ldots & \ldots & \ldots & \ldots & \ldots & \ldots & \ldots & \ldots & \ldots & 212,213\end{array}$

$\begin{array}{llllllllllll}\text { Types of Early Shields } & \ldots & \ldots & \ldots & \ldots & \ldots & \ldots & \ldots & \ldots & \ldots & 214,217\end{array}$

Maennder Pattern from Basilis ..

Bronze Fragments from the Acropolis $\quad \ldots \quad 234,243,245,246,248,251,253$ $256,259,261,262,264,265,268,269$

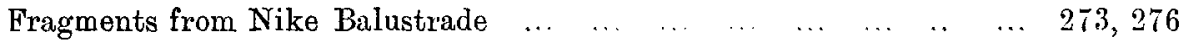

$\begin{array}{lllllllllllll}\text { Section of Balustrade... } & \ldots & \ldots & \ldots & \ldots & \ldots & \ldots & \ldots & \ldots & \ldots & \ldots & \ldots & 278\end{array}$

$\begin{array}{lllllllllll}\text { Terra-Cotta Antefix from Lanuvium... } & \ldots & \ldots & \ldots & \ldots & \ldots & \ldots & \ldots & \ldots & 316\end{array}$

$\begin{array}{llllllllllllll}\text { Section of Antefix } & \ldots & \ldots & \ldots & \ldots & \ldots & \ldots & \ldots & \ldots & & \ldots & \ldots & \ldots & 317\end{array}$

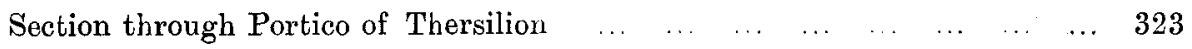

$\begin{array}{llllllllllll}\text { Diagram of Vitruvian Circles } & \ldots & \ldots & \ldots & \ldots & \ldots & \ldots & \ldots & \ldots & \ldots & \ldots & 330\end{array}$ 